Comparison of solution behaviour for three models of pressure-dependent plasticity: A simple analytical example

Alexandrov, S. and Harris, David 2006

MIMS EPrint: 2007.148

Manchester Institute for Mathematical Sciences

School of Mathematics

The University of Manchester

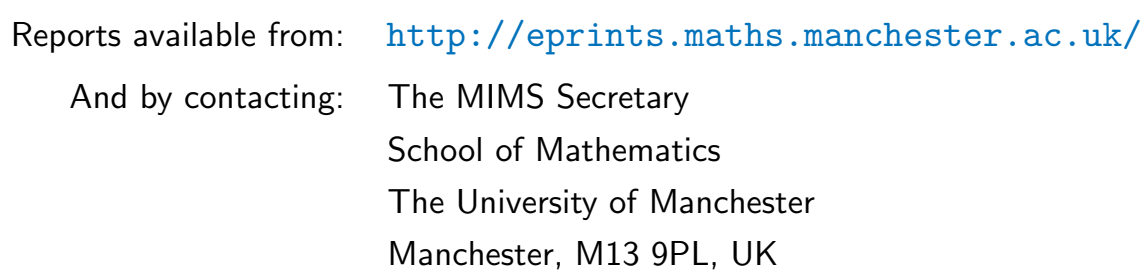

ISSN 1749-9097 


\title{
Comparison of solution behaviour for three models of pressure-dependent plasticity: A simple analytical example
}

\author{
S. Alexandrov ${ }^{\mathrm{a}}, \mathrm{D}$. Harris ${ }^{\mathrm{b}, *}$ \\ ${ }^{a}$ Institute for Problems in Mechanics, Russian Academy of Sciences, 101-1 Prospect Vernadskogo, 119526 Moscow, Russia \\ ${ }^{\mathrm{b}}$ Department of Mathematics, University of Manchester, P.O. Box 88, Manchester M60 1QD, UK
}

Received 11 October 2004; received in revised form 22 December 2005; accepted 28 January 2006

Available online 4 April 2006

\begin{abstract}
The main objective of the present paper is to compare, by means of a problem permitting a closed-form solution, qualitative behaviour of solutions based on three models of pressure-dependent plasticity, the coaxial model, the double-shearing model, and the double-slip and rotation model. The constitutive equations of each model reduce to classical metal plasticity at specific values of input parameters. Nevertheless, the solution behaviour essentially depends on the model chosen, independently of how close the input parameters are to these specific values. In particular, such features of the solutions as non-uniqueness, non-existence and singularity are emphasized. It is concluded that the double-slip and rotation model only retains all features inherent to classical plasticity.
\end{abstract}

C) 2006 Elsevier Ltd. All rights reserved.

Keywords: Rigid/plastic; Pressure-dependent; Coaxial/non-coaxial; Double-slip and spin

\section{Introduction}

Models of pressure-dependent plasticity are used for describing deformation of granular materials, soils and traditional metals (among others Refs. [1-6]). A review of such models is given in Ref. [7]. In contrast to classical metal plasticity, there is no commonly accepted model of pressure-dependent plasticity, though most of such models reduce to classical plasticity at a specific set of parameters. Therefore, it is of interest to apply several models for solving the same problem for understanding the difference in solution behaviour. Several closed-form solutions, including the kinematics of the flow, are available in the literature (for example, among others, Refs. [8-11]). However, these solutions are based on one specific model and therefore do not show the effect of different models.

In this paper, we consider an initial/boundary value problem for rigid/plastic models for the yield and incompressible flow of metals and granular materials and obtain analytic solutions to this problem in the case of three models that have been proposed for materials

\footnotetext{
*Corresponding author.

E-mail address: david.harris@manchester.ac.uk (D. Harris).
}

exhibiting pressure dependence at yield. For completeness, the corresponding solution for classical pressure-independent metal plasticity is also presented [12]. In each case, the Coulomb-Mohr yield criterion, see Eq. (2), is assumed to hold, i.e. the material parameter governing the in-plane pressure dependence is the angle of internal friction $\varphi$ (the case of pressure-independent metal plasticity is then obtained as the special case $\varphi=0$ ). With regard to the flow, each model contains an equation relating the principal axes of stress with the principal axes of the deformation-rate. For the problem that we consider in this paper, it is this equation that distinguishes the three models, which are

(a) co-axial model [13], in which the principal axes of stress and deformation-rate coincide. This, the so-called coaxiality condition, is also one of the governing equations for classical pressure-independent metal plasticity. However, in contrast to classical plasticity, the characteristic directions for stress and velocity do not coincide in the co-axial model.

(b) Double-shearing model [1], in which the equation governing the relationship between the principal axes 
of stress and deformation-rate contains the material derivative of the major principal stress direction, i.e. the model contains the stress rate. This non-coincidence of the principal axes of stress and deformation rate is usually called non-coaxiality and is in contrast to classical plasticity. However, the characteristic directions for stress and velocity coincide, as in classical plasticity.

(c) Double-slip and rotation model [14], in which the stress-rate term in the double-shearing equation mentioned above is replaced by an intrinsic spin $\omega$. In this paper, we only consider a special case of the model in which $\omega$ remains constant, in the general case the model must be considered in the context of a reduced Cosserat continuum. As in the case of the doubleshearing model, the principal directions of stress and deformation-rate tensors do not coincide and the characteristic directions for stress and velocity coincide in this model.

The initial/boundary value problem considered here consists of a planar deformation comprising the simultaneous shearing and expansion/contraction of a hollow cylindrical specimen of material. This combined deformation ensures that there are no rigid regions, i.e. the material is everywhere in a state of yield. Symmetry in the circumferential direction dictates that all quantities are a function of the radial direction only. Both inner and outer cylindrical boundaries expand/contract, the inner boundary also rotates, thereby inducing a shearing motion in the material. Two different regimes may be identified, when the material sticks to the inner boundary and when the material slips at the boundary. In the latter case, there is one boundary condition on the stress and one on the velocity. In the former, there are two boundary conditions on the velocity. For the outer boundary there is one condition on the circumferential velocity.

\section{Systems of equations}

For later convenience, the systems of equations will be written in cylindrical polar coordinates $r \theta z$ under the assumption that the solution is independent of $\theta$ and $z$. The stress equations are

$\frac{\partial \sigma_{r r}}{\partial r}+\frac{\sigma_{r r}-\sigma_{\theta \theta}}{r}=0, \quad \frac{\partial \sigma_{r \theta}}{\partial r}+\frac{2 \sigma_{r \theta}}{r}=0$,

$\left(\sigma_{r r}+\sigma_{\theta \theta}\right) \sin \varphi+\sqrt{\left(\sigma_{r r}-\sigma_{\theta \theta}\right)^{2}+4 \sigma_{r \theta}^{2}}=2 k \cos \varphi$.

Here Eqs. (1) are the equilibrium equations and Eq. (2) is the yield criterion. Also, $\sigma_{r r}, \sigma_{\theta \theta}$ and $\sigma_{r \theta}$ are the components of the stress tensor, $k$ is the cohesion and $\varphi$ is the angle of internal friction. The velocity equations consist of the incompressibility equation

$\frac{\partial u_{r}}{\partial r}+\frac{u_{r}}{r}=0$ and the equation that connects stresses and velocities. The only difference in the models considered lies in this last equation. The equation has the following forms:

$\sin 2 \psi\left(\frac{\partial u_{r}}{\partial r}-\frac{u_{r}}{r}\right)-\cos 2 \psi\left(\frac{\partial u_{\theta}}{\partial r}-\frac{u_{\theta}}{r}\right)=0$

in the case of the co-axial model,

$$
\begin{aligned}
& \sin 2 \psi\left(\frac{\partial u_{r}}{\partial r}-\frac{u_{r}}{r}\right)-\cos 2 \psi\left(\frac{\partial u_{\theta}}{\partial r}-\frac{u_{\theta}}{r}\right) \\
& +\sin \varphi\left(\frac{\partial u_{\theta}}{\partial r}+\frac{u_{\theta}}{r}-2 \frac{\partial \psi}{\partial t}-2 u_{r} \frac{\partial \psi}{\partial r}\right)=0
\end{aligned}
$$

in the case of the double-shearing model, and

$$
\begin{gathered}
\sin 2 \psi\left(\frac{\partial u_{r}}{\partial r}-\frac{u_{r}}{r}\right)-\cos 2 \psi\left(\frac{\partial u_{\theta}}{\partial r}-\frac{u_{\theta}}{r}\right) \\
+\sin \varphi\left(\frac{\partial u_{\theta}}{\partial r}+\frac{u_{\theta}}{r}-2 \omega\right)=0
\end{gathered}
$$

in the case of the double-slip and rotation model. In Eqs. (3)-(6), $u_{r}$ and $u_{\theta}$ are the radial and circumferential velocities, respectively, $\psi$ is the orientation of the major principal stress axis relative to the $r$-axis and $t$ is the time. In the case of Eq. (6), we shall assume $\omega$ to be a constant. In the more general case where $\omega$ is a function of $r$ and $t$, an extra equation governing $\omega$ is required. In the case considered here, $\omega$ is determined by the initial conditions of the problem. Using the angle $\psi$, the yield criterion (2) is automatically satisfied by the substitution

$\sigma_{r r}=\sigma+q \cos 2 \psi, \quad \sigma_{\theta \theta}=\sigma-q \cos 2 \psi, \quad \sigma_{r \theta}=q \sin 2 \psi$,

where

$\sigma=\frac{\sigma_{r r}+\sigma_{\theta \theta}}{2}$ and $q=k \cos \varphi-\sigma \sin \varphi$.

\section{Statement of the problem}

Consider an infinite circular hollow cylinder of internal initial radius $a_{0}$ and external initial radius $b_{0}$ subject to the system of loading consisting of normal and tangential stresses on its internal radius. Due to this system of loading, the cylinder is both expanded/contracted and twisted. It is convenient to introduce a cylindrical polar coordinate system with its $z$-axis coinciding with the axis of symmetry of the cylinder. The current internal radius will be denoted by $a$, then, in the case of incompressible materials, the current external radius is given by

$b=\sqrt{b_{0}^{2}-a_{0}^{2}+a^{2}}$.

The rate of expansion/contraction of the internal radius will be denoted by $\dot{a} \equiv \mathrm{d} a / \mathrm{d} t$. For rate-independent materials, it is not an essential parameter. The external radius is fixed against rotation. Therefore,

$u_{r}=\dot{a}$ 
at $r=a$ and

$u_{\theta}=0$

at $r=b$. One of the stress boundary conditions is

$\sigma_{r r}=-p_{a}<0$

at $r=a . p_{a}$ is given, but its value is not essential for understanding the general features of the solution. The final boundary condition depends on the regime of friction at $r=a$, whether the material is sliding or sticking at the inner boundary. In the case of sliding, the value of the shear stress at $r=a$ is defined by a frictional law and in the present paper, the maximum friction law is adopted. Its analytical representation depends upon the constitutive equations chosen, and it is most conveniently formulated in terms of the angle $\psi$. Without loss of generality, it is possible to assume that $\sigma_{r \theta}>0$. Then, according to Eq. (7),

$\pi / 2>\psi>0$.

The mathematical meaning of the maximum friction law is that the friction surface coincides with an envelope of characteristics such that the solution cannot be extended beyond this surface. In the case of the double-shearing model and the double-slip and rotation model, the characteristics for stress and velocity coincide and are inclined to the $r$-axis at

$\phi=\psi \pm\left(\frac{\pi}{4}+\frac{\varphi}{2}\right)$.

In the problem under consideration, the friction surface makes an angle $\pi / 2$ with the $r$-axis. Putting $\phi=\pi / 2$ in Eq. (14) gives

$\psi=\frac{\pi}{2} \mp\left(\frac{\pi}{4}+\frac{\varphi}{2}\right)$

at the friction surface. The lower sign in Eq. (15) contradicts Eq. (13). Therefore, the frictional boundary condition at sliding for the double-shearing and the double-slip and rotation models is

$\psi=\psi_{w}=\frac{\pi}{4}-\frac{\varphi}{2}$

at $r=a$. In the case of the co-axial model, the orientation of stress characteristics is given by Eq. (14), and the orientation of velocity characteristics by

$\phi=\psi \pm \pi / 4$.

Therefore, it is possible to formulate two possible conditions at $r=a$, one given by Eq. (16), the other by

$\psi=\psi_{w}=\pi / 4$.

Which of these two boundary conditions at sliding will actually occur follows from the structure of the solution. In Fig. 1 the directions of the major principal stress, velocity characteristics and stress characteristics are shown at a generic point of the friction surface. It is possible to distinguish three qualitatively different cases: (i) $\psi>\pi / 4$, this case is shown in Fig. 1a; (ii) $\psi<\pi / 4-\varphi / 2$, this case is shown in Fig. 1b; and (iii) $\pi / 4>\psi>\pi / 4-\varphi / 2$, this case is

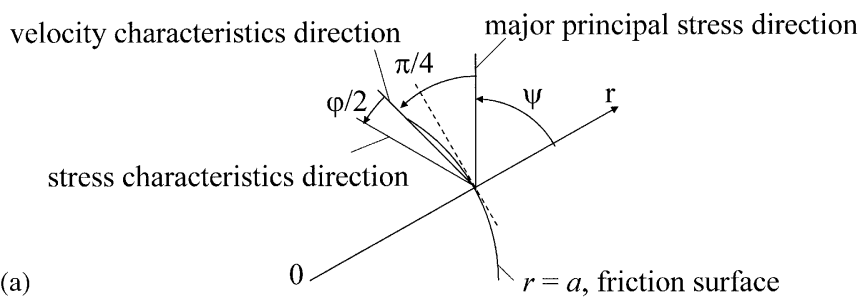

(a)

(b)

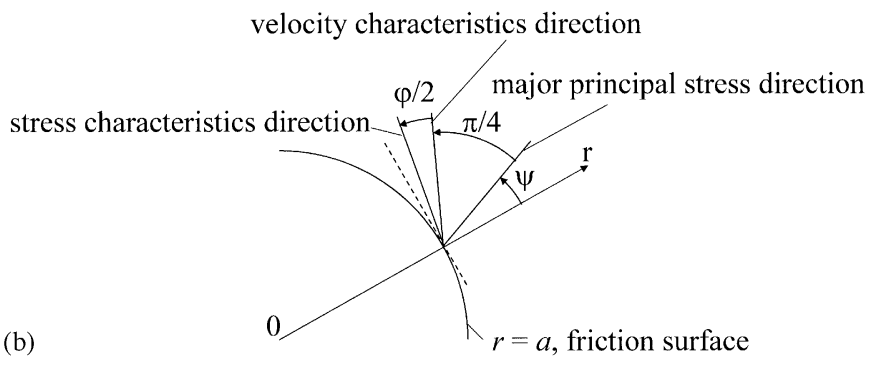

velocity characteristics direction

(c)

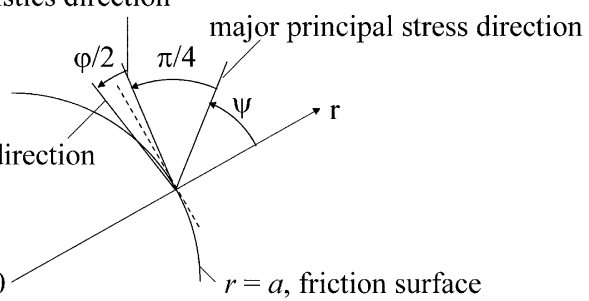

Fig. 1. (a) Illustration of the maximum friction law in case (i). (b) Illustration of the maximum friction law in case (ii). (c) Illustration of the maximum friction law in case (iii).

shown in Fig. 1c. It is seen from Fig. 1a that only a velocity characteristic envelope can coincide with the friction surface. Therefore, in this case, condition (18) holds. It follows from Eq. (7) that for large $\psi$, but within range (13), $\sigma_{\theta \theta}$ is significantly larger than $\sigma_{r r}$. Therefore, it is reasonable to expect that the case (i) corresponds to expansion. It is seen from Fig. 1b that only a stress characteristic envelope can coincide with the friction surface. Therefore, in this case, condition (16) holds. It follows from Eq. (7) that $\sigma_{r r}$ is significantly larger than $\sigma_{\theta \theta}$. Therefore, it is reasonable to expect that the case (ii) corresponds to contraction. Finally, for the case depicted in Fig. 1c, either condition (16) or (18) may represent the maximum friction law. Case (iii) may correspond to either expansion or contraction. The correspondence between the cases (i)-(iii), the two regimes of friction (sticking or sliding), and the two types of deformation (expansion or contraction) will be precisely determined from the solution to the problem.

In the case of sticking, the circumferential velocity is prescribed,

$u_{\theta}=-u_{t}$

at $r=a$, for all models. The assumption $\sigma_{r \theta}>0$ requires $u_{t}>0$. The quantity $u_{t}$ can be regarded as the circumferential velocity of points of the tool surface assuming that the tool is an expanding/contracting and rotating rod 
inserted into the hole of the cylinder. The rate of rod expansion/contraction is indeed equal to $\dot{a}$.

The models under consideration are history-independent. Hence, to understand general features it is sufficient to consider the solutions at one instant, i.e. for a specific geometry. In particular, $b$ and $a$ may be treated as independent variables in spite of Eq. (9). The latter equation should be used only if the process of deformation for a specific specimen needs to be studied. An exception is the transition between the sticking regime and sliding regime where a finite time interval should be considered. However, even in such cases the specific value $b$ is not essential. In what follows, the solutions are mainly understood as instantaneous solutions for given $b$ and $a$.

\section{Solution for stresses}

The general solution to Eq. $(1)^{2}$ is

$\frac{\sigma_{r \theta}}{k}=\frac{\gamma^{2}}{r^{2}}$,

where $\gamma$ is an arbitrary function of $t$, or, more conveniently, $a$. The latter is possible since $\dot{a}$ is a monotonic increasing or decreasing function of $t$ and, therefore, $a$ is a single-valued function of $t$. Combining Eqs. (7) and (20) leads to

$\frac{\sigma_{r r}}{k}=\cot \varphi-\frac{\gamma^{2}(1-\cos 2 \psi \sin \varphi)}{r^{2} \sin 2 \psi \sin \varphi}$,

$\frac{\sigma_{\theta \theta}}{k}=\cot \varphi-\frac{\gamma^{2}(1+\cos 2 \psi \sin \varphi)}{r^{2} \sin 2 \psi \sin \varphi}$.

Substituting Eqs. (20) and (21) into Eq. (1) ${ }^{1}$ gives the following equation for $\psi$ :

$\frac{\partial \psi}{\partial r}=-\frac{\sin 2 \psi}{r(\cos 2 \psi-\sin \varphi)}$.

This equation can be immediately integrated to give

$\frac{r}{a}=\frac{\cos \psi_{a} \tan ^{m} \psi_{a}}{\cos \psi \tan ^{m} \psi}, \quad m=\frac{1-\sin \varphi}{2}$,

where $\psi_{a}$ is the value of $\psi$ at $r=a$ and is a function of $a$. Eq. (23) determines $\psi$ as a function of $r$ and $a$ in implicit form. Substituting $\psi$ in Eq. (21) gives, along with Eq. (20), the solution for stresses in terms of $r$ and $a$ depending on two functions, $\gamma(a)$ and $\psi_{a}(a)$. These functions are to be found from the boundary conditions.

For later convenience, the value of $\psi$ at $r=b, \psi_{b}$, is introduced here. It follows from Eq. (23) that

$\frac{b}{a}=\frac{\cos \psi_{a} \tan ^{m} \psi_{a}}{\cos \psi_{b} \tan ^{m} \psi_{b}}$

\section{Solutions for velocities}

Eq. (3) is involved in all three models and its solution satisfying the boundary condition (10) is

$u_{r}=\frac{\dot{a} a}{r}$.

In the case of the co-axial model, Eq. (4), using Eqs. (22), (23) and (25), can be transformed to

$$
\begin{aligned}
\frac{\partial u_{\theta}}{\partial \psi} & +u_{\theta} \frac{(\cos 2 \psi-\sin \varphi)}{\sin 2 \psi} \\
& =\frac{2 \dot{a}(\cos 2 \psi-\sin \varphi) \cos \psi \tan ^{m} \psi}{\cos \psi_{a} \tan ^{m} \psi_{a} \cos 2 \psi} .
\end{aligned}
$$

The solution to this equation satisfying the boundary condition (11) is

$$
\begin{aligned}
\frac{u_{\theta}}{\dot{a}}= & \frac{2}{\cos \psi_{a} \tan ^{m} \psi_{a} \cos \psi \tan ^{m} \psi} \\
& \times \int_{\psi_{b}}^{\psi} \frac{(\cos 2 \chi-\sin \varphi) \tan ^{2 m} \chi \cos ^{2} \chi}{\cos 2 \chi} \mathrm{d} \chi .
\end{aligned}
$$

In the case of the double-shearing model, Eq. (5), using Eqs. (22), (23) and (25), can be transformed to

$$
\begin{aligned}
& \sin 2 \psi \frac{\partial u_{\theta}}{\partial \psi}+(\cos 2 \psi+\sin \varphi) u_{\theta}-\frac{2 a \dot{a} \sin 2 \psi}{r} \\
& -2 \dot{a} r \sin \varphi \frac{\partial \psi}{\partial a}+\frac{2 a \dot{a} \sin \varphi}{r} \frac{\sin 2 \psi}{(\cos 2 \psi-\sin \varphi)}=0 .
\end{aligned}
$$

Using Eq. (23), it is possible to find the derivative

$$
\frac{\partial \psi}{\partial a}=\frac{\sin 2 \psi}{(\cos 2 \psi-\sin \varphi)}\left[\frac{1}{a}+\frac{\left(\cos 2 \psi_{a}-\sin \varphi\right)}{\sin 2 \psi_{a}} \frac{\mathrm{d} \psi_{a}}{\mathrm{~d} a}\right] .
$$

Substituting Eqs. (23) and (29) into Eq. (28) gives

$$
\begin{aligned}
\frac{\partial u_{\theta}}{\partial \psi}+ & \frac{(\cos 2 \psi+\sin \varphi)}{\sin 2 \psi} u_{\theta} \\
= & \frac{2 \dot{a}}{\cos \psi_{a} \tan ^{m} \psi_{a}(\cos 2 \psi-\sin \varphi)} \\
& \times\left\{\frac{\sin \varphi\left[\sin 2 \psi_{a}+a\left(\cos 2 \psi_{a}-\sin \varphi\right) d \psi_{a} / d a\right]}{2 \tan ^{\sin \varphi} \psi_{a} \cos \psi \tan ^{m} \psi}\right. \\
& \left.+\cos \psi \tan ^{m} \psi(\cos 2 \psi-2 \sin \varphi)\right\} .
\end{aligned}
$$

In the case of the solution to this equation satisfying the boundary condition (11) is

$$
\begin{aligned}
\frac{u_{\theta}}{\dot{a}}= & \frac{\tan ^{m} \psi}{\cos \psi_{a} \tan ^{m+\sin \varphi} \psi_{a} \sin \psi} \\
& \times \int_{\psi_{b}}^{\psi} \frac{\left[\sin \varphi\left[\sin 2 \psi_{a}+a\left(\cos 2 \psi_{a}-\sin \varphi\right) \mathrm{d} \psi_{a} / \mathrm{d} a\right] \tan ^{\sin \varphi} \chi+\tan ^{\sin \varphi} \psi_{a} \sin 2 \chi(\cos 2 \chi-2 \sin \varphi)\right]}{(\cos 2 \chi-\sin \varphi)} \mathrm{d} \chi .
\end{aligned}
$$


The double-slip and rotation model, Eq. (6), using Eqs. (22), (23) and (25), can be transformed to

$$
\begin{aligned}
\frac{\partial u_{\theta}}{\partial \psi} & +\frac{(\cos 2 \psi+\sin \varphi)}{\sin 2 \psi} u_{\theta} \\
& =2 \dot{a}\left(\frac{\Omega \cos \psi_{a} \tan ^{m} \psi_{a} \sin \varphi}{\sin 2 \psi \cos \psi \tan ^{m} \psi}+\frac{\cos \psi \tan ^{m} \psi}{\cos \psi_{a} \tan ^{m} \psi_{a}}\right),
\end{aligned}
$$

where $\Omega=\omega a / \dot{a}$. The solution to this equation satisfying the boundary condition (11) is

$$
\begin{aligned}
\frac{u_{\theta}}{\dot{a}}= & \frac{\tan m \psi}{\sin \psi}\left[\frac{\left(\cos 2 \psi_{b}-\cos 2 \psi\right)}{2 \cos \psi_{a} \tan ^{m} \psi_{a}}\right. \\
& \left.+\Omega \cos \psi_{a} \tan ^{m} \psi_{a} \sin \varphi \int_{\psi_{b}}^{\psi} \frac{\mathrm{d} \chi}{\cos ^{2} \chi \tan ^{2 m} \chi}\right] .
\end{aligned}
$$

\section{Frictional boundary condition}

The solutions obtained in Sections 4 and 5 contain two arbitrary functions of $a$, namely $\gamma$ and $\psi_{a}$. There are two boundary conditions to determine these functions, Eq. (12) and the friction boundary condition. Once $\psi_{a}(a)$ has been found, $\gamma(a)$ can be determined from Eqs. (12) and (21) [1] with no difficulty. Therefore, the key point is the friction boundary condition from which $\psi_{a}(a)$ is to be found. In the case of sliding, $\psi_{a}=\psi_{w}$ and the latter quantity is known due to Eqs. (16) or (18). However, the sliding regime occurs if and only if the shear stress at the friction surface attains a certain level. It will be shown that the solutions based on the models chosen are qualitatively different because of this frictional condition.

Let $u_{0}$ denote $u_{t}$ under the sticking condition and $u_{s l}$ under the sliding condition. In the case of sticking, $u_{t}$ is prescribed and $\psi_{a}$ may be found from the solution. In the case of sliding, $\psi_{a}=\psi_{w}$ is prescribed according to the frictional law Eqs. (16) or (18), and $u_{s l}$ may be found from the solution.

\subsection{The coaxial model}

Eqs. (24) and (27) show that the solution depends on the ratio $b / a$ rather than on $b$ and $a$ separately. Solution (27) gives

$\frac{u_{0}}{\dot{a}}=\frac{2}{\cos ^{2} \psi_{a} \tan ^{2 m} \psi_{a}} \int_{\psi_{a}}^{\psi_{b}} \frac{(\cos 2 \chi-\sin \varphi) \tan ^{2 m} \chi \cos ^{2} \chi}{\cos 2 \chi} \mathrm{d} \chi$

It should be noted that the integral in Eq. (34) is divergent if the point $\psi=\pi / 4$ lies between the points $\psi=$ $\psi_{a}$ and $\psi=\psi_{b}$. Therefore, the points $\psi=\psi_{a}$ and $\psi=\psi_{b}$ lie to one side of the point $\psi=\pi / 4$ and so we consider the sub-intervals $0 \leqslant \psi \leqslant \pi / 4$ and $\pi / 4 \leqslant \psi \leqslant \pi / 2$ separately. The first sub-interval corresponds to case (i), the second to cases (ii) and (iii) introduced in Section 3. The sub-interval $0 \leqslant \psi \leqslant \pi / 4$ may be further sub-divided as follows. If

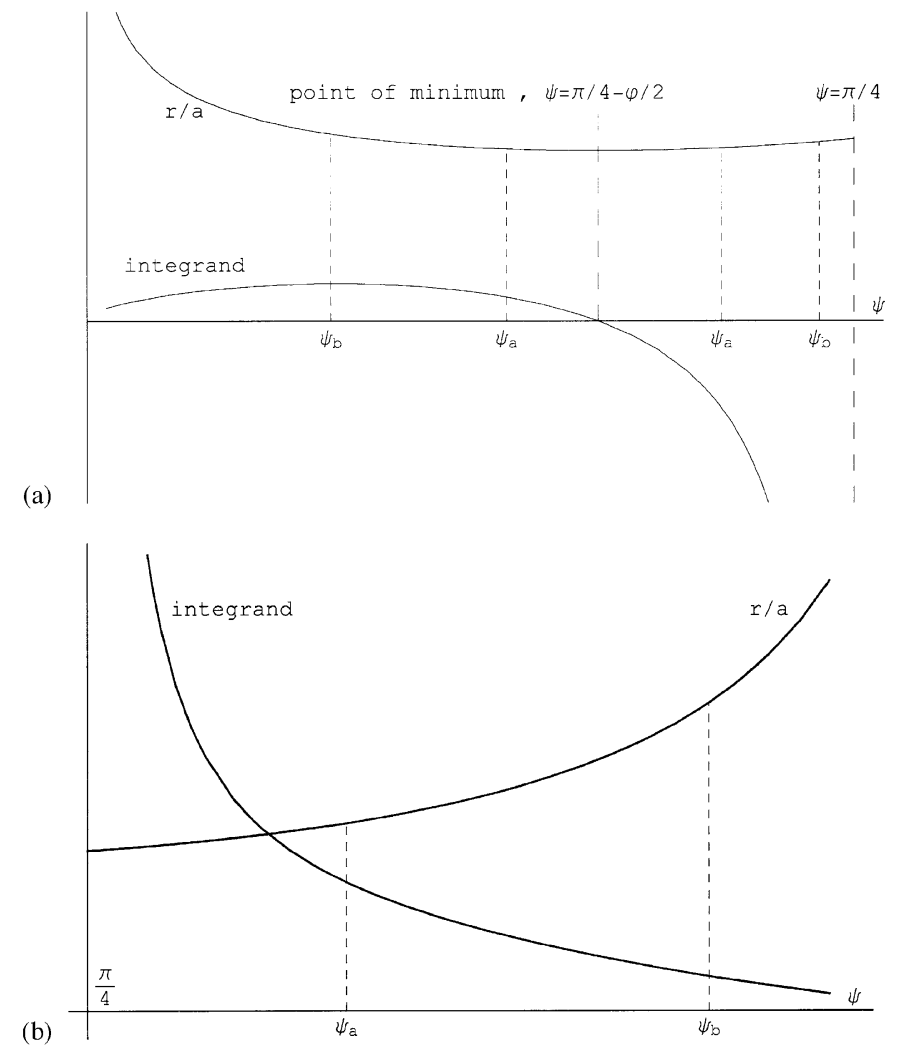

Fig. 2. (a) Coaxial model: $r / a$ and integrand of Eq. (34) as functions of $\psi$, $0 \leqslant \psi \leqslant \pi / 4$. (b) Coaxial model: $r / a$ and the integrand of Eq. (34) as functions of $\psi, \pi / 4 \leqslant \psi \leqslant \pi / 2$.

$\psi_{a}<\pi / 4$ and $\psi_{b}<\pi / 4$, corresponding to cases (ii) and (iii), typical behaviour of the integrand in Eq. (34) and the function $r / a$ defined by Eq. (23) is illustrated in Fig. 2a. It is seen from this figure that the function $r / a$ attains its minimum value at $\psi=\pi / 4-\varphi / 2$. Therefore, we may divide the interval $0 \leqslant \psi \leqslant \pi / 4$ into sub-intervals, $0 \leqslant \psi \leqslant \pi / 4-\varphi / 2$ and $\pi / 4-\varphi / 2 \leqslant \psi \leqslant \pi / 4$, and both $\psi=$ $\psi_{a}$ and $\psi=\psi_{b}$ must lie within one and only one of these sub-intervals. For, if $\psi=\pi / 4-\varphi / 2$ were between $\psi=\psi_{a}$ and $\psi=\psi_{b}$, the function $r / a$ would attain its minimum value between the points $\psi=\psi_{a}$ and $\psi=\psi_{b}$ whereas, by definition, its minimum value is at $\psi=\psi_{a}$ where $r / a=1$. This further division into sub-intervals separates case (ii) from case (iii).

Now consider each of the sub-intervals separately. Since $b>a$, the order of the points $\psi=\psi_{a}$ and $\psi=\psi_{b}$ within each sub-interval must be such as shown in Fig. 2a. Combining the sign of the integrand, the order of the points $\psi=\psi_{a}$ and $\psi=\psi_{b}$, and the structure of Eq. (34), it may be seen that the right-hand side of Eq. (34) is negative in each sub-interval. Since $u_{0}>0$, a solution exists if and only if $\dot{a}<0$. In other words, the interval $0 \leqslant \psi \leqslant \pi / 4$ corresponds to contraction of the cylinder. This confirms the preliminary conclusion concerning the case (ii) made in Section 3 and also clarifies the situation with case (iii). It is seen from Figs. 1b, c and 2a that sliding satisfying condition (16) may occur in both cases (ii) and (iii). 
However, in case (iii), where $\pi / 4-\varphi / 2<\psi<\pi / 4$, the integral in Eq. (34) may approach infinity allowing arbitrarily large values of $u_{t}$. But the curve for $r / a$ depicted in Fig. 2a shows that $b / a$ is small for any $\psi_{a}$ and $\psi_{b}$ within the interval $[\pi / 4-\varphi / 2, \pi / 4]$. In order to illustrate the properties of the solution in the case of contraction combined with twist, numerical calculations have been performed for the value $\varphi=\pi / 6$. The solid curves in Fig. 3 have been obtained in the case (ii) under the sticking condition and show the dependence of the circumferential velocity of the tool, $u_{t} / \dot{a}$, on the ratio $b / a$ at different values of $\psi_{a}$. The dashed curve corresponds to the sliding regime at which $\psi_{a}=\pi / 4-\varphi / 2=\pi / 6$. Therefore, $u_{t} / \dot{a}$ corresponding to this curve is equal to the critical velocity $u_{c r} / \dot{a}$ at which the sticking regime is replaced by the sliding regime. Fig. 2a shows that in case (iii) the maximum possible value of the ratio $b / a,(b / a)_{\max }$, is attained if $\psi_{b}=\pi / 4$. The dependence $(b / a)_{\max }$ on $\psi_{a}$ in the range $\psi_{w}=\pi / 4-\varphi / 2=\pi / 6 \leqslant \psi_{a} \leqslant \pi / 4$ is illustrated in Fig. 4. This maximum value of $b / a$ has been used in calculation of the dependence of $u_{t} / \dot{a}$ on $b / a$ in the case (iii). Under the sticking condition, this dependence (solid curves) is depicted in Fig. 5 at different values of $\psi_{a}$. The dashed curve corresponds to the sliding regime at which $\psi_{a}=\pi / 4-\varphi / 2=\pi / 6$. Therefore, $u_{t} / \dot{a}$ corresponding to this curve is equal to the critical velocity $u_{t} / \dot{a}$ at which

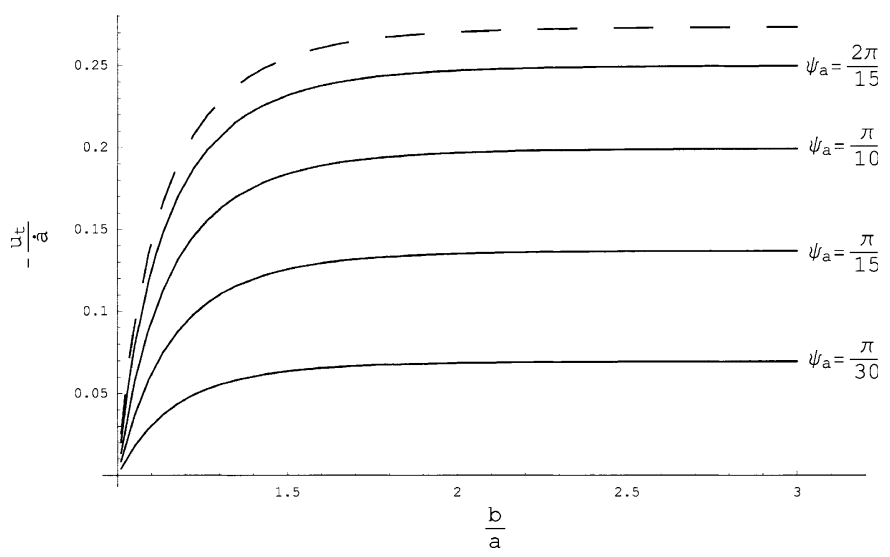

Fig. 3. Coaxial model: circumferential velocity versus $b / a$-contraction.

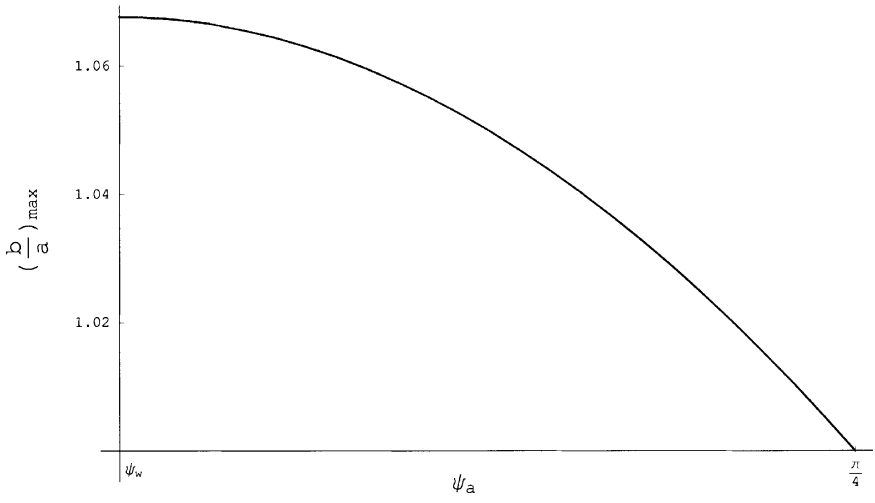

Fig. 4. Coaxial model: maximum of $b / a$ versus $\psi_{a}$.

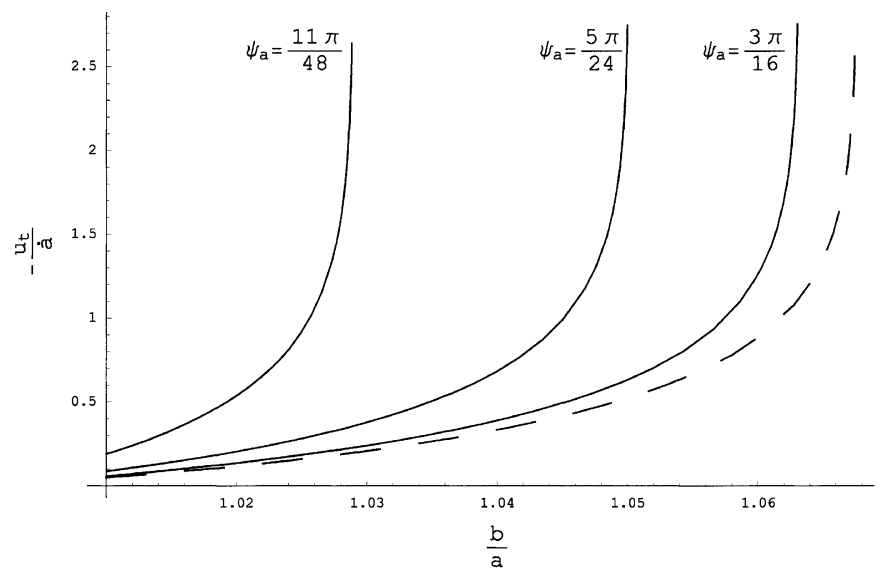

Fig. 5. Coaxial model: circumferential velocity versus $b / a, \pi / 4-\varphi / 2 \leqslant \psi$ $\leqslant \pi / 4$.

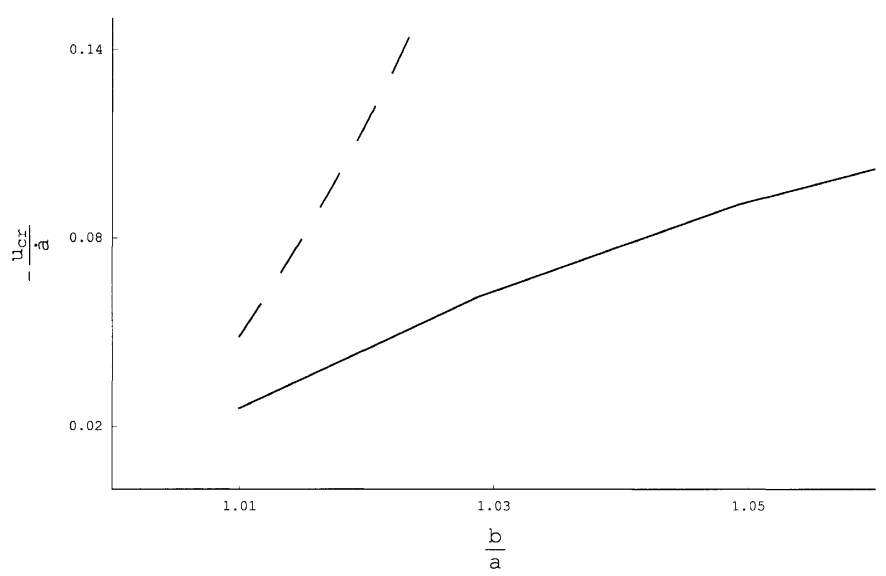

Fig. 6. Coaxial model: transition between sticking and sliding.

sticking is replaced by sliding. However, in contrast to the previous case, for any value of $b / a$ the magnitude of the critical velocity is less than the magnitude of $u_{t} / \dot{a}$ at any $\psi_{a}>\pi / 4-\varphi / 2$. Therefore, sliding is the only possible friction regime in the case (iii). Since the cases (ii) and (iii) are related to the same process, namely contraction with twist, it is necessary to compare the values of $u_{c r} / \dot{a}$ found for each case. This comparison is illustrated in Fig. 6 in the range of $b / a$ where the solution for case (iii) exists. The dashed curve corresponds to the regime (iii) and the solid curve to the regime (ii). It is seen from Fig. 6 that the critical velocity in the regime (ii) is lower than the critical velocity in the regime (iii) in the entire range of $b / a$. Therefore, sliding starts when the critical velocity in the case (ii) is reached and thus the case (iii) never occurs.

In the case (i), combining the sign of the integrand, the order of the points $\psi=\psi_{a}$ and $\psi=\psi_{b}$ (Fig. 2b), and the structure of Eq. (34), it is possible to see that its right-hand side is positive at $\psi_{a}>\pi / 4$ and $\psi_{b}>\pi / 4$. Since $u_{0}>0$, we must conclude that a solution exists if and only if $\dot{a}>0$. Hence the interval $\pi / 4 \leqslant \psi \leqslant \pi / 2$ corresponds to the expansion of the cylinder. This confirms the preliminary conclusion concerning the case (i) made in Section 3. In this 


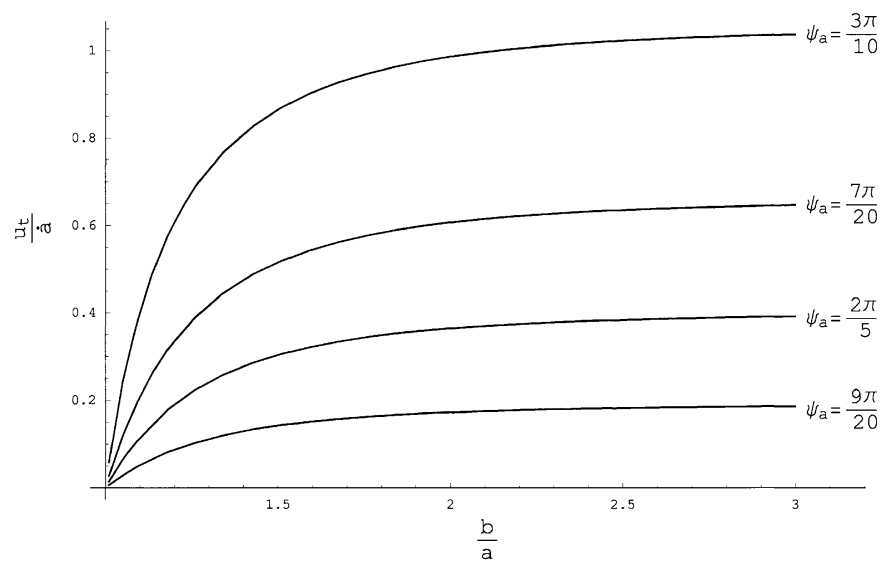

Fig. 7. Coaxial model: circumferential velocity versus $b / a$ - expansion.

process of the expansion combined with twist, it is seen from Figs. $1 \mathrm{a}$ and $2 \mathrm{~b}$ that sliding satisfying condition (18) might occur according to the frictional law. However, since the integral in Eq. (34) is divergent if $\psi_{a}=\pi / 4$, sticking is the only possible friction regime in this case, which makes a significant qualitative difference from the process of the contraction combined with twist. The dependence $u_{t} / \dot{a}$ on $b / a$ is shown in Fig. 7. It is seen from this figure that the circumferential velocity of the tool is relatively low even at $\psi_{a}=3 \pi / 10$. This means that a very significant increase in the velocity occurs within a very small interval of $\psi_{a}$ near the point $\psi_{a}=\pi / 4$. For instance, the ratio $u_{t} / \dot{a}$ is about 8 when the difference between $\psi_{a}$ and $\pi / 4$ is of the order of $10^{-8}$.

The radial distribution of the circumferential velocity at $b / a=2$ is shown in Fig. 8 for the contraction and in Fig. 9 for the expansion. The dashed curve in Fig. 8 corresponds to the sliding regime.

\subsection{The double-shearing model}

In the case of this model, solution (31) gives

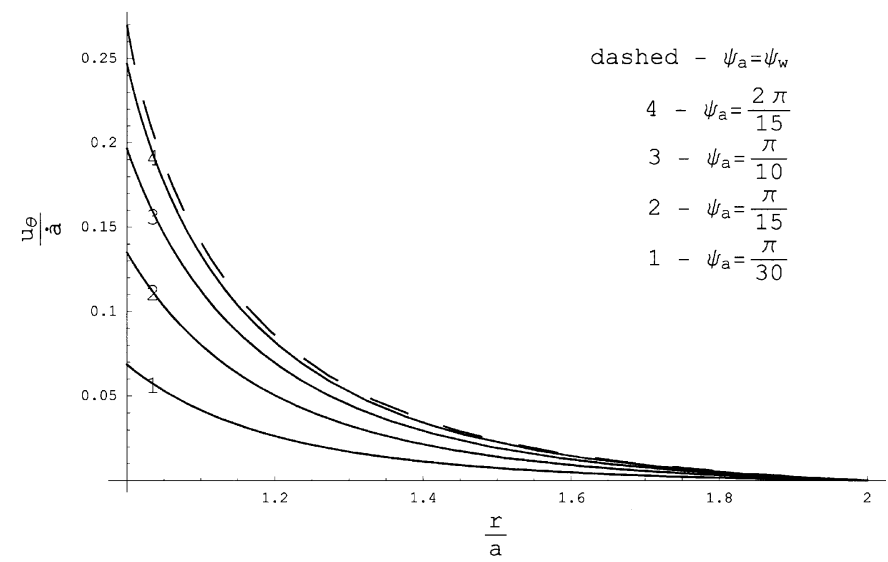

Fig. 8. Coaxial model: circumferential velocity versus $r / a$ - contraction.

Eq. (23) gives

$$
\begin{aligned}
\frac{u_{s l}}{\dot{a}}= & \frac{2}{h \cos \varphi} \int_{\psi_{w}}^{\psi_{b}} \\
& \times \frac{\left\{\sin \varphi \cos \varphi \tan ^{\sin \varphi} \chi+h \sin 2 \chi(\cos 2 \chi-2 \sin \varphi)\right\}}{(\cos 2 \chi-\sin \varphi)} \mathrm{d} \chi .
\end{aligned}
$$

Here and in what follows

$h=\left(\frac{\cos \varphi}{1+\sin \varphi}\right)^{\sin \varphi}$.

It follows from Eqs. (24) and (36) that in the case of the double-shearing model the solution at sliding depends on the ratio $b / a$ rather than on $b$ and $a$ separately. Note that it is not true in general, since $\mathrm{d} \psi_{a} / \mathrm{d} a$ may not vanish under the sticking condition and, therefore, Eq. (35) may involve $a$. In such cases, it is more convenient to adopt $b / a$ and $a$ as the independent variables instead of $b$ and $a$.

It is easy to see that the denominator and the numerator of the integrand in Eq. (36) vanish at $\chi=\psi_{w}$. Applying l'Hospital rule it is possible to obtain

$$
\begin{aligned}
\frac{u_{\theta}}{\dot{a}}= & \frac{2}{\sin 2 \psi_{a} \tan ^{\sin \varphi} \psi_{a}} \\
& \times \int_{\psi_{a}}^{\psi_{b}} \frac{\left[\sin \varphi \tan ^{\sin \varphi} \chi\left[\sin 2 \psi_{a}+a\left(\cos 2 \psi_{a}-\sin \varphi\right) \mathrm{d} \psi_{a} / \mathrm{d} a\right]+\tan ^{\sin \varphi} \psi_{a} \sin 2 \chi(\cos 2 \chi-2 \sin \varphi)\right]}{(\cos 2 \chi-\sin \varphi)} \mathrm{d} \chi
\end{aligned}
$$

At sliding $\psi_{a}=\psi_{w}$ is prescribed according to Eq. (16) and $u_{s l}$ may be found from the solution. For the sticking condition, Eq. (35) is an ordinary differential equation for $\psi_{a}$ and a difficulty with this equation is that there is no initial condition for $\psi_{a}$, unless sliding occurs at the beginning of the process. In the latter case, $\psi_{a}=\psi_{w}=$ $\pi / 4-\varphi / 2=$ const and, therefore, $\mathrm{d} \psi_{a} / \mathrm{d} a=0$. Substituting these conditions into Eq. (35) and eliminating $m$ using

$$
\begin{aligned}
& \lim _{\chi \rightarrow \psi_{w}}\left\{\frac{\sin \varphi \cos \varphi \tan ^{\sin \varphi} \chi+h \sin 2 \chi(\cos 2 \chi-2 \sin \varphi)}{(\cos 2 \chi-\sin \varphi)}\right\} \\
& \quad=h \cos \varphi .
\end{aligned}
$$

Therefore, Eq. (36) where $\psi_{b}$ should be eliminated using Eq. (24) determines the finite value of $u_{s l}$. The kinematic condition for sliding to occur is $u_{t}>u_{s l}$. The critical value of the velocity $u_{t}$ at which transition between the sliding 


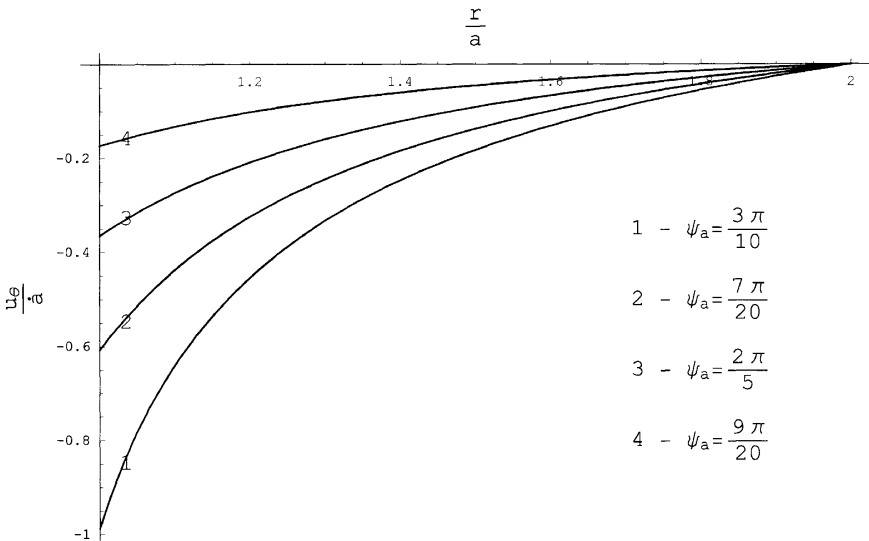

Fig. 9. Coaxial model: circumferential velocity versus $r / a$-expansion.

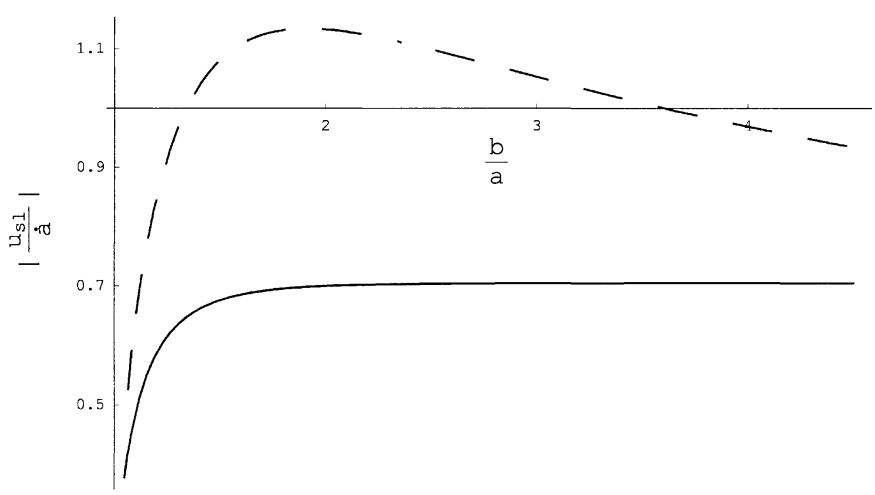

Fig. 10. Double-shearing model: transition between sticking and sliding.

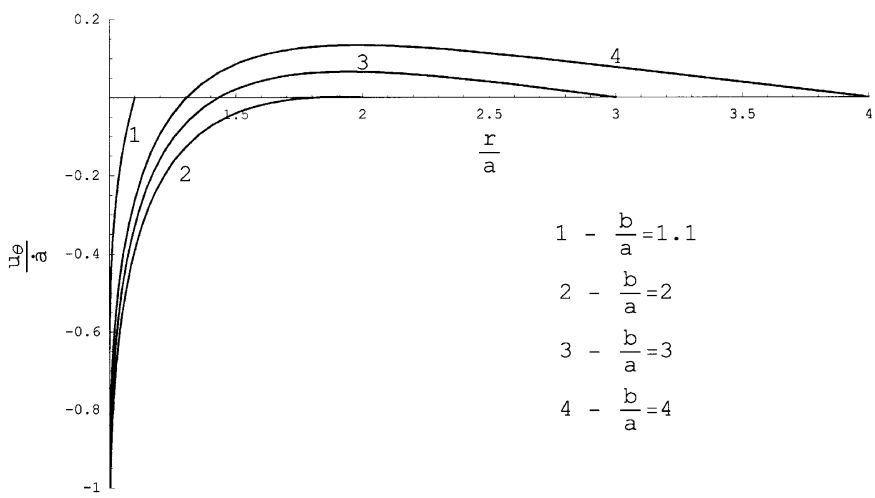

Fig. 11. Double-shearing model: circumferential velocity versus $r / a-$ expansion.

and sticking regimes may occur, $u_{c r}$, is determined by the equation $u_{c r}=u_{s l}$ and has been found at $\varphi=\pi / 6$ (Fig. 10). The dashed curve corresponds to the expansion, $\pi / 4-\varphi / 2 \leqslant \psi<\pi / 2$, and the solid curve to the contraction, $0<\psi \leqslant \pi / 4-\varphi / 2$. The radial distribution of the circumferential velocity is shown in Fig. 11 in the case of the expansion and in Fig. 12 in the case of the contraction for different ratios $b / a$.

Assume that $u_{t}>u_{c r}$ at the beginning of the process when $a=a_{0}, u_{t}=u_{c r}$ at $a=a_{c r}$ and then $u_{t}<u_{c r}$. In such a

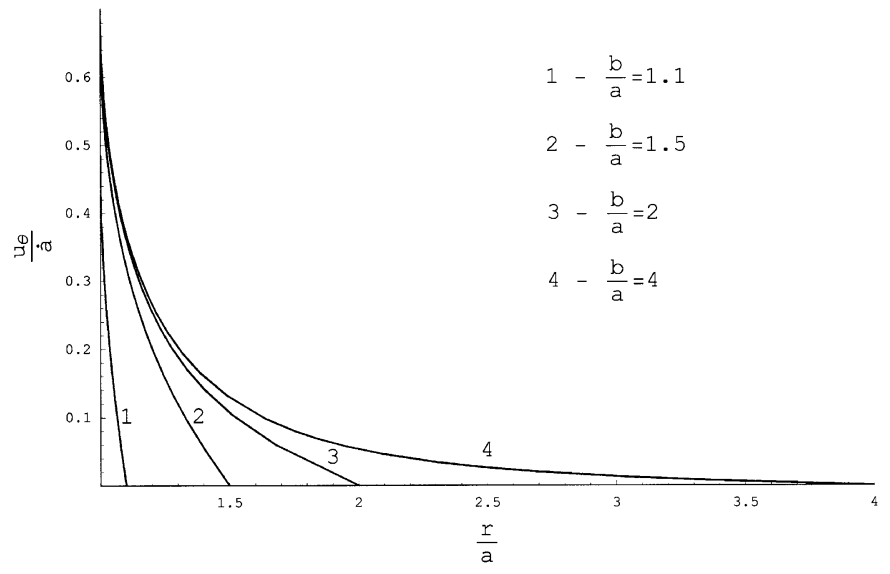

Fig. 12. Double-shearing model: circumferential velocity versus $r / a$ contraction.

process, the regime of sticking must replace the regime of sliding at $a=a_{c r}$ Then Eq. (35) considered as the differential equation for $\psi_{a}(a)$ may be solved for $a \geqslant a_{c r}$ in the case of the expansion or $a \leqslant a_{c r}$ in the case of the contraction together with the boundary condition

$\psi_{a}=\psi_{w} \quad$ at $a=a_{c r}$.

As in the case of Eq. (36), the integrand in Eq. (35) reduces to the expression $0 / 0$ at $\psi_{a}=\psi_{w}$ and $a=a_{c}$. However, in contrast to Eq. (36), it is a function of two variables, $\chi$ and $a$ for $a \geqslant a_{c r}$ in the case of the expansion or $a \leqslant a_{c r}$ in the case of the contraction. To find the asymptotic behaviour of $\psi_{a}(a)$ in the vicinity of the point $\psi_{a}=\psi_{w}$ and $a=a_{c r}$, the functions involved in the numerator and the denominator of the integrand in Eq. (35) can be expanded in a series with respect to $\chi$ around $\chi=\psi_{w}$. The result is

$\tan ^{\sin \varphi} \chi=h\left[1+2\left(\chi-\psi_{w}\right) \tan \varphi\right]+o\left(\chi-\psi_{w}\right)$, $\sin 2 \chi(\cos 2 \chi-2 \sin \varphi)=-\cos \varphi \sin \varphi-2\left(\chi-\psi_{w}\right)$

$$
+o\left(\chi-\psi_{w}\right) \text {, }
$$

$\cos 2 \chi-\sin \varphi=-2\left(\chi-\psi_{w}\right) \cos \varphi+o\left(\chi-\psi_{w}\right)$.

Neglecting terms of order of $o\left(\chi-\psi_{w}\right)$ Eq. (35), using Eq. (39) in the vicinity of the point $a=a_{c r}$, may be rewritten in the form

$$
\begin{aligned}
\frac{u_{t}}{\dot{a}}= & \frac{2}{h \cos \varphi} \int_{\psi_{a}}^{\psi_{w}+\psi_{\delta}}\left[\lambda(a)+\frac{\mu(a)}{\left(\chi-\psi_{w}\right)}\right] \mathrm{d} \chi \\
& +I_{1}(a) \quad \text { as } \psi_{a} \rightarrow \psi_{w},
\end{aligned}
$$

where

$$
\begin{aligned}
\lambda(a)= & h\left\{\cos \varphi+\left[2 \tan \varphi \frac{\mathrm{d} \psi_{a}}{\mathrm{~d} a}\left(\cos \varphi+a_{c r} \sin \varphi \frac{\mathrm{d} \psi_{a}}{\mathrm{~d} a}\right)\right]\right. \\
& \left.\times\left(a-a_{c r}\right)\right\}+o\left(a-a_{c r}\right), \\
\mu(a)= & a_{c r} h \sin \varphi\left(\frac{\mathrm{d} \psi_{a}}{\mathrm{~d} a}\right)^{2}\left(a-a_{c r}\right)+o\left(a-a_{c r}\right) .
\end{aligned}
$$

In Eq. (41), the derivatives are understood to be calculated at $a=a_{c r}\left(\right.$ or $\left.\psi_{a}=\psi_{w}\right)$. Also, $\psi_{\delta} \ll 1$ is a constant such that 
$\psi_{w} \leqslant \psi_{a} \leqslant \psi_{w}+\psi_{\delta}$ and $I_{1}(a)$ is a non-singular function of $a$ resulting from Eq. (35) after integration from $\psi_{w}+\psi_{\delta}$ to $\psi_{b}$. After integration equation (40) takes the form

$\frac{u_{t}}{\dot{a}}=-\frac{2}{h \cos \varphi} \mu(a) \ln \left|\psi_{a}-\psi_{w}\right|+I_{2}(a)$,

where $I_{2}(a)$ is the non-singular term consisting of $I_{1}(a)$ and the non-singular term obtained after integration in Eq. (40). It follows from Eq. (41) that the singular term in Eq. (42) approaches zero for any function $\psi_{a}(a)$ with a bounded derivative at $\psi_{a}=\psi_{w}$. Therefore, its contribution to the magnitude of $u_{t}$ is negligible. However, some derivatives of $u_{t}$ may be controlled by the singular term near the point $\psi_{a}=\psi_{w}$. In fact, assuming that $\mathrm{d} \psi_{a} / \mathrm{d} t \neq 0$ at $\psi_{a}=\psi_{w}$ and differentiating Eq. (42) using Eq. (41) gives

$\frac{\mathrm{d}}{\mathrm{d} a}\left(\frac{u_{t}}{\dot{a}}\right)=-2 a_{c r} \tan \varphi\left(\frac{\mathrm{d} \psi_{a}}{\mathrm{~d} a}\right)^{2} \ln \left(\psi_{a}-\psi_{w}\right)$

to leading order. To avoid infinity in this equation, it is necessary to put $d \psi_{a} / d t=0$ at $\psi_{a}=\psi_{w}$. The latter condition is sufficient to get a bounded value for the second derivative of $u_{t} / \dot{a}$. However, the condition that the third derivative is bounded requires that $\mathrm{d}^{2} \psi_{a} / \mathrm{d} t^{2}=0$ at $\psi_{a}=\psi_{w}$. This process may be continued to the derivative of any order. The same result can be obtained directly from Eq. (35). The denominator of the integrand is independent of $a$. Therefore, as $\psi_{a} \rightarrow \psi_{w}$, the derivative of any order contains a singular term in the form

$\int_{\psi_{a}}^{\psi_{b}} \frac{\Phi(a)}{(\cos 2 \chi-\sin \varphi)} \mathrm{d} \chi$,

where $\Phi(a)$ may include derivatives of $\psi_{a}$ as $\psi_{a} \rightarrow \psi_{w}$. In the original expression (35), the function $\Phi(a) \rightarrow 0$ as $a \rightarrow$ $a_{c r}$ (or $\psi_{a} \rightarrow \psi_{w}$ ) leading to a converging integral independently of the value of $\mathrm{d} \psi_{a} / \mathrm{d} a$ at $\psi_{a}=\psi_{w}$. This condition is however not satisfied when Eq. (44) is the singular term involved in a derivative of $u_{t} / \dot{a}$ and it is necessary to put $\mathrm{d}^{n} \psi_{a} / \mathrm{d} a^{n}=0$ at $\psi_{a}=\psi_{w}$ for obtaining bounded values of $\mathrm{d}^{n}\left(u_{t} / \dot{a}\right) / \mathrm{d} a^{n}$ and $\mathrm{d}^{n+1}\left(u_{t} / \dot{a}\right) / \mathrm{d} a^{n+1}$. Therefore, it must be concluded that transition from sliding to sticking with a continuous function $\psi_{a}(a)$ is possible if and only if the function $u_{t} / \dot{a}$ satisfies certain restrictions. In particular, its derivatives of order $n$ and higher do not exist (i.e. they approach infinity) at $\psi_{a}=\psi_{w}$. The specific number $n$ is determined by the behaviour of the function $\psi_{a}(a)$ in the vicinity of the point $a=a_{c r}$. The larger the value of $n$, the higher the order of the derivative of $\psi_{a}(a)$ vanishing at $a=a_{c r}$ must be. On the other hand, $u_{t} / \dot{a}$ is an independent function determined by the motion of the tool. Since this motion may be incompatible with the aforementioned behaviour of the function $\psi_{a}$, the solution for transition between the sliding and sticking regimes may not exist. On the other hand, it is possible to show that the solution at $u_{t}=u_{s l}$ is not unique. To this end, a solution under the sticking condition is found below assuming that $u_{t}=u_{s l}$. It is sufficient to consider the initial instance when $a=a_{c r}$. In Eq. (35), $u_{0}$ should be replaced with $u_{s l}$. Then, this equation may be treated in the following manner. Prescribe any value of $\psi_{a}$ within the interval $(\pi / 4-\varphi / 2, \pi / 2)$ in the case of the expansion or within the interval $(0, \pi / 4-\varphi / 2)$ in the case of the contraction. Eq. (35) should be now solved for $a \mathrm{~d} \psi_{a} / \mathrm{d} a$. If such a solution exists, the corresponding value of $\psi_{a}$ can be used as the boundary condition to solve Eq. (35) and by the choice of this value the sticking regime occurs. The existence of the solution is illustrated in Figs. 13 and 14 at $\varphi=\pi / 6$. In Fig. 13, for several ratios $b / a$, the curves determine the couples $\psi_{a}$ and $a \mathrm{~d} \psi_{a} / \mathrm{d} a$ such that $u_{t}=u_{s l}$ in the expansion where $u_{s l}$ is shown in Fig. 10 by the dashed curve. Fig. 14 shows similar results for the compression. In this case, the difference in curves for different $b / a$ is relatively small because $u_{s l}$ is almost constant at $b / a>2$ (Fig. 10). Therefore, two curves only are shown, the dashed curve corresponds to $b / a=1.5$ and the solid curve to $b / a=4$.

Thus in the case of the double-shearing model there are two types of non-uniqueness where $u_{t}=u_{s l}$ : (i) one can obtain the solution either under the sliding condition or under the sticking condition, (ii) in the case of sticking, it is possible to get an infinite number of solutions by selecting different couples $\psi_{a}$ and $a \mathrm{~d} \psi_{a} / \mathrm{d} a$ satisfying Eq. (35) at $a=a_{c r}$ (possible couples are illustrated in Figs. 13 and 14).

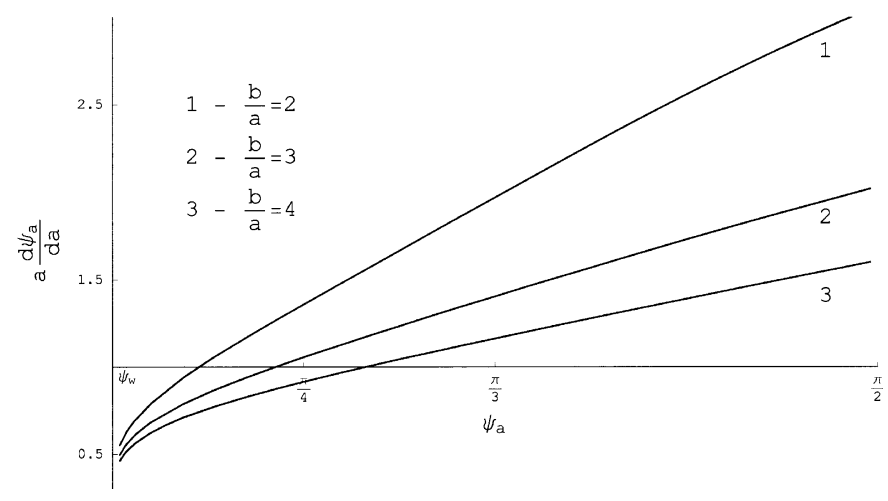

Fig. 13. Double-shearing model: stress-rate versus stress - expansion.

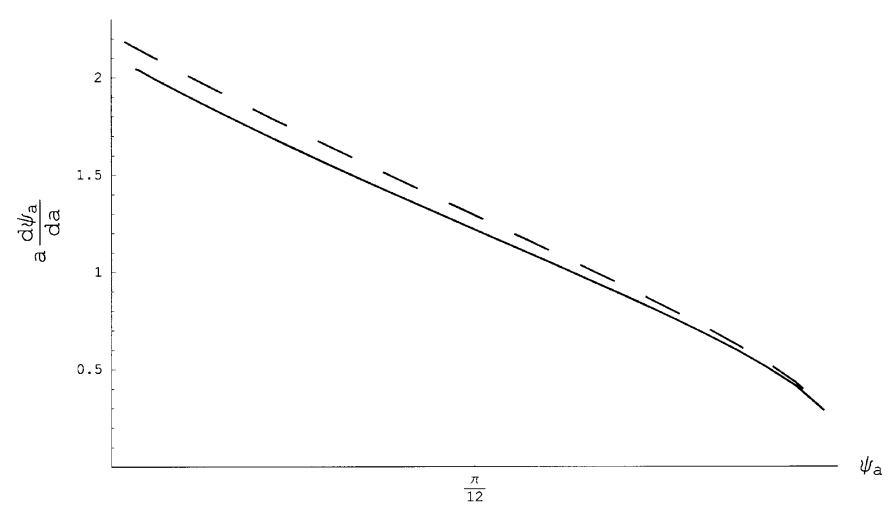

Fig. 14. Double-shearing model: stress-rate versus stress - contraction. 
If $u_{t} \neq u_{s l}$, the solution is not unique because there is no initial condition for Eq. (35) considered as the ordinary differential equation for $\psi_{a}$, which is somehow similar to the case (ii) when $u_{t}=u_{s l}$.

\subsection{Double-slip and rotation model}

Eq. (24) and (33) show that the solution depends on the ratio $b / a$ and $a$ (through $\Omega$ ). In the case of the double-slip and rotation model, Eq. (33) combined with the frictional boundary condition gives

$$
\begin{aligned}
\frac{u_{0}}{\dot{a}}= & \frac{\tan ^{m} \psi}{\sin \psi}\left[\frac{\left(\cos 2 \psi_{a}-\cos 2 \psi_{b}\right)}{2 \cos \psi_{a} \tan ^{m} \psi_{a}}+\Omega \cos \psi_{a} \tan ^{m} \psi_{a} \sin \varphi\right. \\
& \left.\times \int_{\psi_{a}}^{\psi_{b}} \frac{\mathrm{d} \chi}{\cos ^{2} \chi \tan ^{2 m} \chi}\right] .
\end{aligned}
$$

It is seen from this equation that the integral is not singular at $\psi_{a}=\psi_{w}$, in contrast to both the double-shearing and coaxial models. Therefore, for any given value of $\Omega$, specific values of $a_{c r}$ and $u_{c r}<\infty$ can be found from Eq. (45) at $\psi_{a}=\psi_{w}$. If $u_{t}<u_{c r}$, the unique solution at

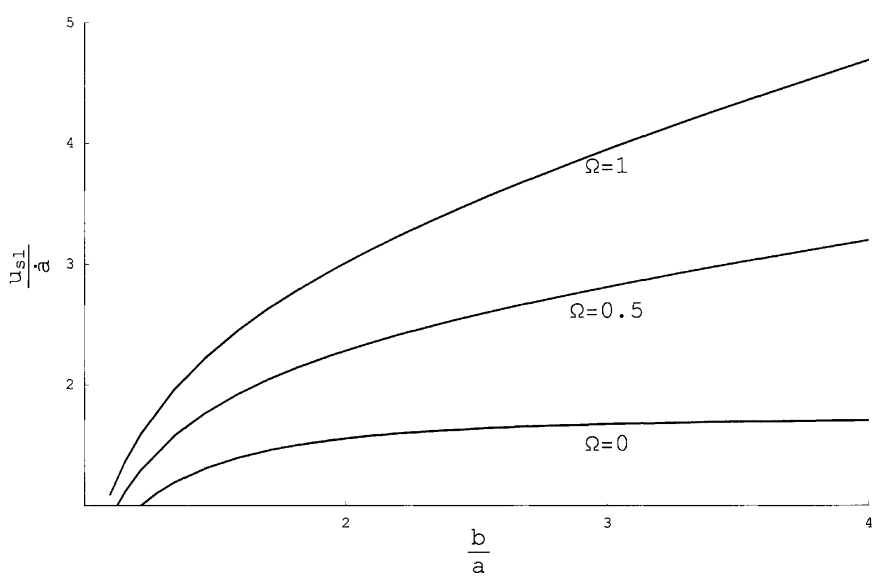

Fig. 15. Double-slip and rotation model, circumferential velocity—sliding and expansion.

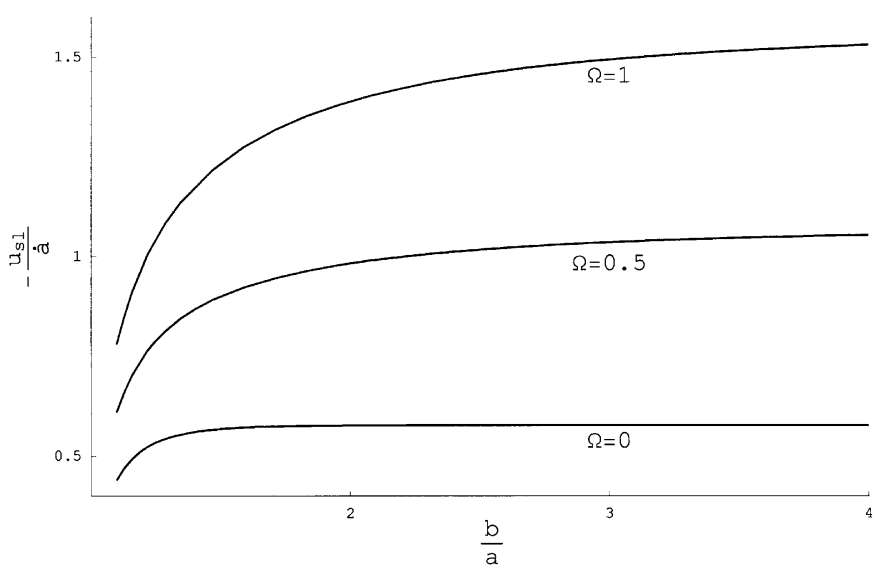

Fig. 16. Double-slip and rotation model, circumferential velocity—sliding and contraction.

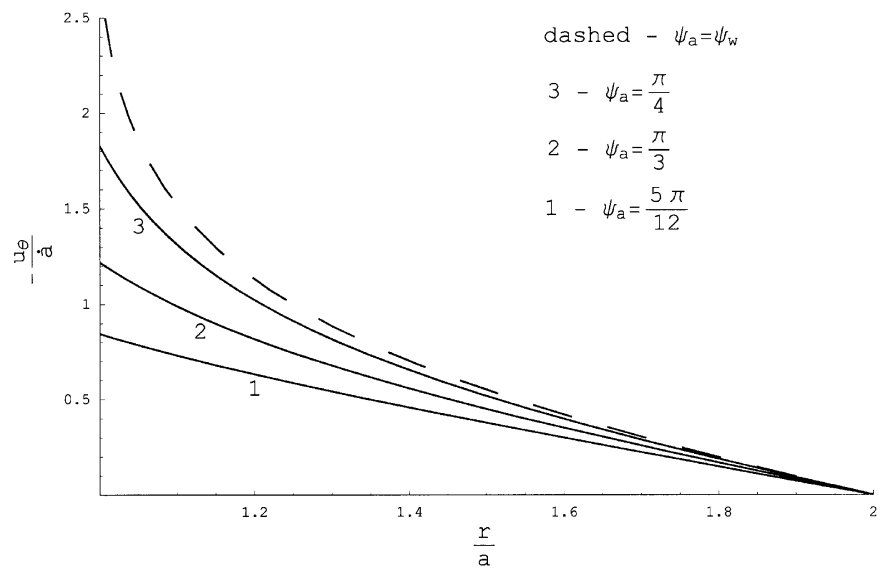

Fig. 17. Double-slip and rotation model: circumferential velocity versus $r / a$ expansion.

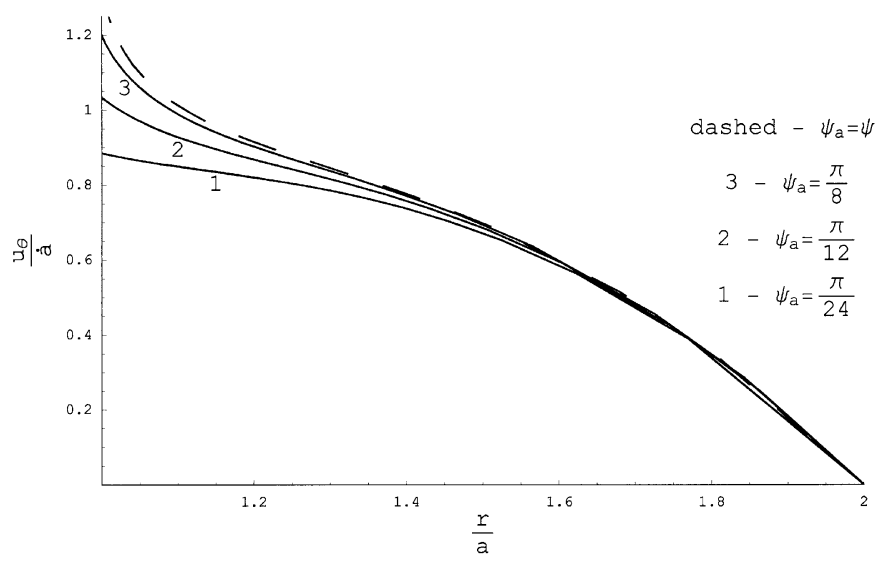

Fig. 18. Double-slip and rotation model: circumferential velocity versus $r / a$ - contraction.

sticking exists and if $u_{t}>u_{c r}$ the unique solution at sliding exists. At $u_{t}=u_{c r}$, transition between the regimes occurs such that $\psi_{a}$ is a continuous function of $a$. At $\varphi=\pi / 6$, the variation of $u_{s l}$ with $b / a$ for different $\Omega$ is shown in Fig. 15 for the extension of the cylinder, $\psi_{w} \leqslant \psi<\pi / 2$, and in Fig. 16 for the contraction of the cylinder, $0<\psi \leqslant \psi_{w}$. At $b / a=2$, the radial distribution of the circumferential velocity obtained by means of Eqs. (24) and (33) is illustrated in Fig. 17 for the expansion and in Fig. 18 for the contraction. The dashed curves in these figures correspond to the sliding regime.

\section{Solution of pressure-independent plasticity}

The solution based on the equations of rigid/perfectly plastic material with a pressure-independent yield criterion has been proposed in Ref. [12]. The system of equations consists of the equilibrium equations (1), the yield condition in the form

$\sqrt{\left(\sigma_{r r}-\sigma_{\theta \theta}\right)^{2}+4 \sigma_{r \theta}^{2}}=2 k$ 
the incompressibity Eq. (3) and the co-axiality condition (4). Eq. (46) may be obtained from Eq. (2) at $\varphi=0$, and $\psi$ in this section has the same meaning as before and $k$ is the shear yield stress. The maximum friction law is defined by Eq. (18). Even though the co-axiality condition is satisfied, an essential difference from the co-axial model is that in the case under consideration the characteristics for stresses and velocities coincide, which is a property of the double-shearing and double-slip and rotation model.

The yield condition (46) is satisfied by the substitution

$\sigma_{r r}-\sigma_{\theta \theta}=2 k \cos 2 \psi, \quad \sigma_{r \theta}=k \sin 2 \psi$.

As before, the distribution of the shear stress is given by Eq. (20). Using Eqs. (20), (25) and (47), the solution to Eq. (4) satisfying Eq. (11) can be found in the form

$\frac{u_{\theta}}{\dot{a}}= \pm \frac{r a}{\gamma^{2}}\left(\frac{\sqrt{b^{4}-\gamma^{4}}}{b^{2}}-\frac{\sqrt{r^{4}-\gamma^{4}}}{r^{2}}\right)$.

Here and in what follows the upper sign corresponds to the contraction, $0<\psi \leqslant \pi / 4$, and the lower sign to the expansion, $\pi / 4 \leqslant \psi<\pi / 2$.

In the case of sliding, it follows from Eqs. (18), (20) and (47) that $\gamma=a$. Substituting this equation into Eq. (48) at $r=a$ gives

$\frac{u_{s l}}{\dot{a}}=\mp \sqrt{1-\left(\frac{a}{b}\right)^{4}}$.

In the case of sticking, it follows from Eqs.(19) and (48) that

$\frac{u_{t}}{\mp \dot{a}}=\frac{a^{2}}{\gamma^{2}}\left(\sqrt{1-\left(\frac{\gamma}{b}\right)^{4}}-\sqrt{1-\left(\frac{\gamma}{a}\right)^{4}}\right)$.

This equation determines $\gamma$ at given $a$ and $b$. In particular, it follows from Eq. (50) that

$0<\gamma \leqslant a$.

At given $a$ and $b$, the right-hand side of Eq. (50) is a monotonically increasing function of $\gamma$ in the interval $0<\gamma \leqslant a$. Therefore, within this interval the maximum is attained at $\gamma=a$. Hence taking into account Eq. (51), it is possible to conclude that the solution under the sticking condition does not exist for $u_{t}>u_{c r}$ where $u_{c r}$ is determined from Eq. (50) at $\gamma=a$. Comparing with Eq. (49) shows that $u_{c r}=u_{s l}$. Therefore, in the case of classical plasticity, both regimes, sliding and sticking, may occur and the solution is unique for both processes, the expansion and contraction.

\section{Singularity in velocity fields}

In the case of sliding, the velocity fields in the solutions based on the double-shearing model, double-slip and rotations model and the classical plasticity model are singular. To show this it is necessary to find the shear strain rate. In the problem under consideration it is defined by

$2 \xi_{r \theta}=\frac{\partial u_{\theta}}{\partial r}-\frac{u_{\theta}}{r}$.

The first term may be rewritten in the form

$\frac{\partial u_{\theta}}{\partial r}=\frac{\partial u_{\theta}}{\partial \psi} \frac{\partial \psi}{\partial r}$.

It follows from Eq. (30), using Eqs. (37), that for the double-shearing model the derivative $\partial u_{\theta} / \partial \psi$ is bounded and, in general, is not zero at $\psi=\psi_{w}$. The same conclusion may be drawn from Eq. (32) for the double-slip and rotation model. On the other hand, it follows from Eq. (22) that $\partial \psi / \partial r \rightarrow \infty$ as $\psi \rightarrow \psi_{w}$. Therefore, it is clear from Eqs. (53) and (52) that $\xi_{r \theta} \rightarrow \infty$ as $\psi \rightarrow \psi_{w}$ and thus the velocity field is singular. In the vicinity of the point $\psi=\psi_{w}$ (or $r=a$ ), Eq. (22) can be rewritten in the form

$\frac{\partial \psi}{\partial r}=\frac{1}{2 a\left(\psi-\psi_{w}\right)}$

to leading order. The solution to Eq. (54) satisfying the condition $\psi=\psi_{w}$ at $r=a$ is

$\psi-\psi_{w}= \pm \frac{\sqrt{r-a}}{\sqrt{a}}$.

Substituting Eq. (55) into Eq. (54) gives

$\frac{\partial \psi}{\partial r}=O\left(\frac{1}{\sqrt{r-a}}\right)$.

Then, it follows from Eqs. (52) and (53) that

$\xi_{r \theta}=O\left(\frac{1}{\sqrt{r-a}}\right)$.

Since $\xi_{r \theta} \rightarrow \infty$ as $r \rightarrow a$, its magnitude controls the behaviour of the equivalent strain rate, $\xi_{e q}$, in the vicinity of the friction surface,

$\xi_{e q}=O\left(\frac{1}{\sqrt{r-a}}\right)$.

This is also a general property of the equations of classical plasticity [15]. In particular, in the problem under consideration, Eq. (58) can be obtained from Eq. (48) by inspection. Alexandrov and Lyamina [16] have found the behaviour (58) in the case of plane-strain deformation of materials obeying the double-shearing model.

In the case of the co-axial model, Eq. (26) shows that $\partial u_{\theta} / \partial \psi$ is proportional to $(\cos 2 \psi-\sin \varphi)$. Therefore, the product on the right in Eq. (53) is bounded at the friction surface, as follows from Eqs. (22) and (58) does not hold for this model.

\section{Summary, conclusions and discussion}

The planar quasi-static initial/boundary value problem comprising simultaneous ring shear and expansion/contraction of a hollow cylinder filled with incompressible rigid/perfectly plastic material in which the material is 
everywhere in a state of yield is solved. Four different kinematic models are considered, namely pressure-independent metal plasticity and three pressure-dependent models, coaxial, double-shearing and double-slip and rotation. The equations governing the flow comprise Eqs. (1)-(3), together with one of the Eqs. (4)-(6). The boundary conditions for the problem fall into two categories, firstly, Eqs. (10) and (12) prescribe the radial velocity and radial component of stress at $r=a$, and equation Eq. (11) prescribes the circumferential velocity component at $r=b$. Secondly, two kinematic regimes at the inner boundary are considered, namely sliding and sticking, each of which has an associated stress or velocity boundary condition. Thus in the sliding regime, either Eq. (16) holds (in the case of the double-shearing and double slip and rotation model) or one of Eqs. (16) and (18) hold (in the case of the coaxial model). For the sticking regime, Eq. (19) holds.

The stress field also imposes one of two possible kinematic conditions, namely, expansion or contraction of the hollow cylinder. For pressure-independent plasticity, the case of expansion corresponds to $\frac{1}{4} \pi \leqslant \psi \leqslant \frac{1}{2} \pi$, while contraction corresponds to $0 \leqslant \psi \leqslant \frac{1}{4} \pi$, where $\psi$ denotes the angle of inclination of the algebraically greater principal stress direction to the radial direction. For the coaxial model, expansion corresponds to $\frac{1}{4} \pi \leqslant \psi \leqslant \frac{1}{2} \pi$, while contraction corresponds to $0 \leqslant \psi \leqslant \frac{1}{4} \pi-\frac{1}{2} \varphi$. For the doubleshearing and double-slip and rotation models, contraction corresponds to $0 \leqslant \psi \leqslant \frac{1}{4} \pi-\frac{1}{2} \varphi$, while expansion corresponds to $\frac{1}{4} \pi-\frac{1}{2} \varphi \leqslant \psi \leqslant \frac{1}{2} \pi$.

It is well known that, for quasi-static flow, the stress equilibrium equations uncouple from the kinematic equations and are determinate, i.e. may be solved independently of the kinematic equations, provided there are sufficient stress boundary conditions. The kinematic equations may then be solved using the stress solution. For the problem considered here, the stress field, for all of the models, is given by Eqs. (20), (21) and (23).

Turning to the velocity field, the radial velocity component satisfies Eq. (3), together with the boundary condition Eq. (10) and, for all three models, the solution is given by Eq. (25). The remaining kinematic equation, governing the circumferential velocity $u_{\theta}$ is Eq. (26) for the coaxial model, Eq. (28) for the double-shearing model and Eq. (32) for the double slip and rotation model. The corresponding solutions, satisfying the velocity boundary condition equation (11), are Eq. (27) for the coaxial model, Eq. (31) for the double-shearing model and Eq. (33) for the double slip and rotation model. The stress boundary condition Eq. (12) and the stress representation Equation $(21)^{1}$ determine $\gamma$ in terms of $a$ and the value of $\psi(a)$. Finally, Eqs. (27), (31) or (33) are used to determine a relationship between $u_{\theta}(a)$ and $\psi(a)$, and this is given by Eq. (34) for the coaxial model, Eq. (35) for the doubleshearing model and Eq. (45) for the double-slip and rotation model. Then, in the sticking regime, $u_{\theta}(a)$ is prescribed and $\psi(a)$ is determined in terms of $u_{\theta}(a)$, while in the case of sliding, $\psi(a)$ is prescribed by Eqs. (16) or (18) and $u_{\theta}(a)$ is then determined in terms of $\psi(a)$. For each model, the equation relating $u_{\theta}(a)$ and $\psi(a)$ involves an integration over the interval $[\psi(a), \psi(b)]$ or $[\psi(b), \psi(a)]$.

For the coaxial model, the interval $[0, \pi / 2]$ may be decomposed into three sub-intervals, $[0, \pi / 4-\varphi / 2]$, $[\pi / 4-\varphi / 2, \pi / 4],[\pi / 4, \pi / 2]$, the integral is singular at $\psi=$ $\pi / 4$ and the interval of integration must itself be a subinterval of one of these three sub-intervals. Corresponding to contraction, in the sub-interval $[0, \pi / 4-\varphi / 2], \psi_{b}<\psi_{a}$ and sticking occurs if $\psi_{a}<\pi / 4-\varphi / 2$ while sliding occurs at $\psi_{a}=\pi / 4-\varphi / 2$. The sub-interval $[\pi / 4-\varphi / 2, \pi / 4]$ never occurs. Corresponding to expansion, in the sub-interval $[\pi / 4-\pi / 2], \psi_{a}<\psi_{b}$, only sticking occurs, there can be no sliding, and the velocity component $u_{\theta}$ is unbounded at $r=a$ if $\psi=\pi / 4$.

For the double-shearing model, in the sliding regime, Eq. (36) determines $u_{\theta}(a)$. In this case the integrand in Eq. (36) is indeterminate but the integral exists and $u_{\theta}(a)$ is finite. In the sticking regime, Eq. (35) may be regarded as a linear first-order differential equation for $\psi(a)$, however, in the sticking regime, the initial value of $\psi(a)$ is not prescribed unless it happens that at this instant the regime is changing from sliding to sticking. Thus, in general, in the sticking regime there are infinitely many solutions, one for each pair of values $\left(\psi(\mathrm{a}), a \mathrm{~d} \psi_{\mathrm{a}} / \mathrm{da}\right)$. The transition from sliding to sticking with a continuous $\psi(a)$ places restrictions on the derivatives and it is possible that a solution may not exist.

The solution for pressure-independent metal plasticity is simpler than the solutions for the other models in that all quantities may be found explicitly in terms of $r$, whereas for the other models, $\psi$ must be obtained implicitly in terms of $r$ and the velocity $u_{\theta}$ is then obtained, via an integral representation of the solution, as a function of $\psi$, i.e. $u_{\theta}$ is also obtained implicitly as a function of $r$. Now, for both the coaxial and the double-shearing models there are values of $\psi$ for which the integral representation for $u_{\theta}$ is singular for physically realizable values of $\psi$, whereas for the double-slip and rotation the integral is never singular for such values. In this sense, the double-slip and rotation model behaves in a manner similar to pressure-independent metal plasticity. Also, for both pressure-independent metal plasticity and the double-slip and rotation model, the solutions in the sticking and sliding regimes exist and are unique, and for both models there is a critical value of $u_{\theta}$ which governs the transition between the two regimes and at which $\psi(a)$ is continuous. On the other hand, as noted above, the coaxial model has problems with existence of solutions for physically realizable values of $\psi$, for example there is no solution in the interval $[\pi / 4-\varphi / 2, \pi / 4]$. Also, the double-shearing model has non-uniqueness of solutions in the sticking regime, it is possible that the transition from sliding to sticking may not exist, there are restrictions on the function $u_{t} / \dot{a}$ as a function of $a$ in order for a solution to exist. This indicates that the further investigation of the full double-slip and rotation model in the context of a 
reduced Cosserat continuum may well prove to be worthwhile and this will be the subject of future papers.

Finally, we note that the models considered here, being based upon incompressibility and a pressure-dependent yield condition, are necessarily of the non-associated flow rule type. The next step in the analysis of these models is to introduce a general non-associated flow rule which will enable dilatation/consolidation to be incorporated into the model. This will complicate the equations since, in this case, the two velocity equations become coupled, even in the case of constant dilatation/consolidation and recourse will have to be made to numerical methods of integration. In reality, the situation is even more complicated since the yield condition for such materials involves the porosity which itself depends on the velocity field. Hence in this case the stress and velocity equations are coupled and hence must be solved simultaneously. One method of simplifying the analysis when compressibility is incorporated is to adopt an associated flow rule. A review of such models can be found in Ref. [17] and models of this class are usually used to analyse and design processes in powder metallurgy. The disadvantage of an associated flow rule is that there is only one parameter to model both the pressure-dependence and the degree of compressibility, while real materials require two parameters. In the case of pressure-independent plasticity, the analysis for an associated flow rule is presented in Section 7. It is of interest to note that all three models considered here reduce to the same associated flow rule. The solution of the equations governing the models when compressibility is included will be a subject of the further research.

\section{Acknowledgements}

The main part of this work was done while the authors participated in the programme Granular and ParticleLaden Flows at the Isaac Newton Institute for Mathematical Sciences (Cambridge, UK). S.A. acknowledges support from the Russian Foundation for Basic Research (Grant 05-01-00153) and the program State Support of Leading Scientific Schools, Russia (Grant NSH1849.2003.1).

\section{References}

[1] Spencer AJM. A theory of the kinematics of ideal soils under plane strain conditions. Journal of the Mechanics and Physics of Solids 1964;12:337-51.

[2] de Josselin de Jong G. The double-sliding, free-rotating model for granular assemblies. Geotechnique 1971;21:155-63.

[3] Anand L. Plane deformations of ideal granular materials. Journal of the Mechanics and Physics of Solids 1983;31:105-22.

[4] Kao AS, Kuhn HA, Spitzig WA, Richmond O. Influence of superimposed hydrostatic pressure on bending fracture and formability of a low carbon steel containing globular sulfides. Transactions of the ASME Journal of Engineering Materials and Technology 1990;112:26-30.

[5] Harris D. Constitutive equations for planar deformations of rigidplastic materials. Journal of the Mechanics and Physics of Solids 1993;41:1515-31.

[6] Harris D. A unified formulation for plasticity models of granular and other materials. Proceedings of the Royal Society Series A 1995;450: $37-49$.

[7] Ostrowska-Maciejewska J, Harris D. Three-dimensional constitutive equations for rigid/perfectly plastic granular materials. Mathematical Proceedings of the Cambridge Philosophical Society 1990;108: 153-69.

[8] Pemberton CS. Flow of imponderable granular materials in wedgeshaped channels. Journal of the Mechanics and Physics Solids 1965;13:351-60.

[9] Marshall EA. The compression of a slab of ideal soil between rough plates. Acta Mechanica 1967;3:82-92.

[10] Spencer AJM. Plastic flow past a smooth cone. Acta Mechanica 1984;54:63-74.

[11] Alexandrov S, Lyamina E. Plane-strain compression of material obeying the double-shearing model between rotating plates. International Journal of Mechanical Sciences 2003;45:1505-17.

[12] Alexandrov S, Richmond O. Frictional effects in the modified Couette flow of solids. In: Pietrzyk M, Kusiak J, Majta J, Hartley P, Pillinger I, editors. Proceedings of the Eigth International Conference on Metal Forming. Rotterdam: Balkema; 2000. p. 723-8.

[13] Hill R. The mathematical theory of plasticity. Oxford: Clarendon Press; 1950.

[14] Harris D, Grekova EF. A hyperbolic well-posed model for the flow of granular materials. Journal of Engineering 2005;52:107-35.

[15] Alexandrov S, Richmond O. Singular plastic flow fields near surfaces of maximum friction stress. International Journal of Non-Linear Mechanics 2001;36:1-11.

[16] Alexandrov S, Lyamina E. Singular solutions for plane plastic flow of pressure-dependent materials. Doklady-Physics, A Journal of the Russian Academy of Sciences 2002;47:308-11.

[17] Druyanov B. Technological mechanics of porous bodies. New-York: Clarendon Press; 1993. 